\title{
Model Predictive Control of Linear Periodic Systems - A Unified Framework Including Control of Multirate and Multiplexed Systems
}

\author{
Ravi Gondhalekar, Colin N. Jones
}

\begin{abstract}
State-feedback model predictive control (MPC) of discrete-time linear periodic systems with possibly timedependent state and control input dimension is considered. States and inputs are subject to hard, mixed, polytopic constraints. It is described how discrete-time linear systems, both time-invariant and periodic, with multirate or multiplexed control inputs can be modeled as such periodic systems. This makes linear periodic systems with possibly time-dependent dimensions a unified, coherent and succinct state-space modeling framework for a large variety of control problem for linear plants, periodic or non. In this paper it is shown how important theoretical results for state-feedback MPC of constrained linear time-invariant (LTI) systems are conceptually equivalent to what is required for linear periodic systems. Specifically the determination of (maximum) periodic controlled and positively invariant sets and the solution of reverse periodic discretetime algebraic Riccati equations are considered indispensable. A general definition, and a method for the determination, of maximum periodic controlled and positively invariant sets are proposed here. Thus least-restrictive, strongly feasible MPC problems resulting in infinite-horizon optimal state-feedback control laws are designed. The proposed methods are applied to a multirate twin-actuator nano-positioning system.
\end{abstract}

Keywords: Model predictive control; Linear periodic systems; Multirate systems; Multiplexed systems; Hard constraints

\section{INTRODUCTION}

MPC theory for constrained discrete-time LTI systems is well established $[10,14,15]$. In this paper well-known LTIMPC theory is generalized to linear periodic systems. Of particular and novel interest is the need to develop methods which accommodate periodically time-dependent state and control input dimension. It is shown that the basic ingredients necessary to enforce strong feasibility and provide a priori guarantees of stability and optimality are conceptually identical to those typically employed in MPC of usual LTI systems. These are the ability to determine (maximum) controlled and positively invariant sets [3] and solutions to Riccati equations [2], and enforce these as terminal constraints and costs, respectively [15]. The solution of reverse periodic discrete-time algebraic Riccati equations with possibly timedependent dimensions has been studied [5,21]. The determination of suitably general periodic invariant sets has not. An appropriate definition for (maximum) periodic controlled

Ravi Gondhalekar is with the Frontier Research Base for Global Young Researchers and Department of Mechanical Engineering, Osaka University, Japan. He is funded through the Program of Promotion of Environmental Improvement to Enhance Young Researchers' Independence under the Special Coordination Funds for Promoting Science and Technology, Japan Ministry of Education, Culture, Sports, Science and Technology. ravi.gondhalekar@wakate.frc.eng.osaka-u.ac.jp

Colin N. Jones is with the Automatic Control Laboratory, Department of Electrical Engineering, Swiss Federal Institute of Technology in Zurich (ETHZ), Switzerland. cjones@ee.ethz.ch and positively invariant sets is proposed in this paper, and a method for their determination is presented.

MPC of linear periodic systems has been considered ([4] and references therein). However, current methods do not accommodate time-dependent dimensions, and the use of elliptic terminal sets and linear matrix inequality stability conditions generally results in restrictive and suboptimal control laws. Furthermore the methods are theoretically somewhat distinct form LTI-MPC methods. LTI systems are linear periodic systems with period length one. Thus LTI-MPC methods should be a specific instance of periodic MPC methods. The methods developed in this paper lead to least-restrictive and infinite-horizon optimal control laws, and are true generalizations of LTI-MPC methods. A further approach is RepetitiveMPC (RMPC) [11]. There a linear periodic system is lifted to an LTI system and LTI-MPC methods applied. Unfortunately the lifting increases the effective system step-size to an entire period length.This introduces delays in responding to disturbances and changes in reference input.

The motivation for considering systems with timedependent dimensions is control law synthesis for systems with asynchronous control inputs. In particular multirate and multiplexed control inputs are considered. In input-multirate systems each control input channel may have a unique update period [18]. In this paper linear periodic systems with time-dependent dimensions are employed as a modeling framework for input-multirate linear plants, assuming all input rates are integer multiples of some base period. Use of periodic systems to model multirate LTI systems was reported in $[1,16,18]$. However, those have time-invariant dimensions. These have two main drawbacks for control law synthesis. First, employing time-invariant dimensions does not encode the input timing behavior within the model. By utilizing time-dependent dimensions the asynchronous input timing can be embedded into the plant model and abstracted away. This alleviates the need to enforce the timing from within the controller (Sec. IV). Thus controller design becomes more transparent and can be performed using (nearly) standard methods (Sec. III). Second, minimal system realizations generally require time-dependent dimensions $[6,20]$. Thus models with time-invariant dimensions are generally not minimal and result in unnecessary computational complexity. This is especially so for MPC, where excessive model dimensions accumulate over multiple prediction steps.

In input-multiplexed systems the control inputs channels are updated asynchronously in ordered sequence. In this paper this is again modeled as a linear periodic system. Inputmultiplexed control was considered in $[12,17]$ for increasing 
the performance to complexity ratio of MPC. An equivalence to periodic systems was mentioned but not fully exploited. The models employed there are non-minimal, which may lead to a waste of processor clock-cycles. The determination of invariant sets was ignored in [12]. Thus [12] is not directly applicable to constrained MPC, because the use, either explicit or implicit, of invariant sets is critical for enforcing basic properties like strong feasibility and stability $[3,15]$. Set invariance is addressed here. Note that the multiplexed MPC strategy of $[12,17]$ is different than just MPC of input-multiplexed systems. The unified MPC framework proposed in this paper incorporates the first stage of the twostage multiplexed controller design procedure of $[12,17]$. The second stage synthesizes low-complexity suboptimal MPC control laws and is unrelated to the methods of this paper.

Notation: The real number set is denoted by $\mathbb{R}\left(\mathbb{R}_{0}\right.$ : non-negative. $\mathbb{R}_{+}$: strictly positive), the set of non-negative integers $\mathbb{N}\left(\mathbb{N}_{+}:=\mathbb{N} \backslash\{0\}\right)$, the set of consecutive nonnegative integers $\{j, \ldots, k\}$ by $\mathbb{N}_{j}^{k}$. Denote by $\bmod : \mathbb{N} \times$ $\mathbb{N}_{+} \rightarrow \mathbb{N}, \bmod (i, j):=\min _{k \in \mathbb{N}}\{i-k j \mid i-k j \geq 0\}$ the remainder. Denote by $I_{n} \in\{0,1\}^{n \times n}$ the identity matrix, by $0_{\{n, m\}} \in\{0\}^{n \times m}$ the zero matrix and by 0 without subscript the zero matrix with dimension deemed obvious by context. Element $j$ of a vector $a$ is denoted by $a_{[j]}$. For matrices $A$ and $B$ of equal dimension, matrix inequalities $A\{<, \leq, \geq,>\} B$ hold component-wise. The set of non-empty subsets of a set $\mathbb{X}$, i.e. the power set less $\emptyset$, is denoted by $2^{\mathbb{X}}$. A sequence of elements $x_{i} \in \mathbb{X} \forall i \in \mathbb{N}_{j}^{k}$ is denoted by $\left\{x_{i} \in \mathbb{X}\right\}_{i=j}^{k}$. Let $\psi_{(i, k)}$ denote the future value of variable $\psi$ at step $i+k$, as predicted from step $i$. For compact notation $\psi_{(i, 0)} \equiv \psi_{i}$.

\section{Motivation for Linear Periodic Systems}

Definition 1: A constrained discrete-time LTI system is defined by dynamics (1) and constraints (2) with step index $i \in \mathbb{N}$, state $x \in \mathbb{R}^{n}, n \in \mathbb{N}_{+}$, control input $u \in \mathbb{R}^{m}$, $m \in \mathbb{N}_{+}, A \in \mathbb{R}^{n \times n}, B \in \mathbb{R}^{n \times m}, E \in \mathbb{R}^{r \times n}, G \in \mathbb{R}^{r \times m}$, $W \in \mathbb{R}^{r}$, and $r \in \mathbb{N}_{+}$:

$$
\begin{gathered}
x_{i+1}=A x_{i}+B u_{i}, \\
E x_{i}+G u_{i} \leq W .
\end{gathered}
$$

Definition 2: A constrained discrete-time linear periodic system is defined by dynamics (3) and constraints (4) with step index $i \in \mathbb{N}$, period length $p \in \mathbb{N}_{+}$, inter-period step index $j \in \mathbb{N}_{0}^{p-1}$, state $\bar{x}_{i} \in \mathbb{R}^{n_{j}}$, control input $\bar{u}_{i} \in \mathbb{R}^{m_{j}}$, and $\left[n_{j} \in \mathbb{N}_{+}\right] \wedge\left[m_{j} \in \mathbb{N}_{+}\right] \wedge\left[A_{j} \in \mathbb{R}^{n_{\bmod (j+1, p)} \times n_{j}}\right] \wedge$

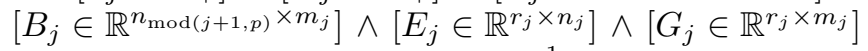
$\wedge\left[W_{j} \in \mathbb{R}^{r_{j}}\right] \wedge\left[r_{j} \in \mathbb{N}_{+}\right] \forall j \in \mathbb{N}_{0}^{p-1}$ :

$$
\begin{aligned}
& \bar{x}_{i+1}=A_{j} \bar{x}_{i}+B_{j} \bar{u}_{i} \quad, \quad j=\bmod (i, p), \\
& E_{j} \bar{x}_{i}+G_{j} \bar{u}_{i} \leq W_{j} .
\end{aligned}
$$

The unusual and critical aspect of linear periodic systems according to Definition 2 is that the periodic state and input dimensions are time-dependent, not only the system matrices. The methods of this paper are in concept applicable when $m_{j}=0$ or $n_{j}=0$ for some values of $j$. To avoid notational abuses or obfuscated notation Definition 2 describes only periodic systems with strictly positive dimensions.
Throughout this paper, periodic states and inputs $(\bar{x}, \bar{u})$ are differentiated from their time-invariant cousins $(x, u)$ by an over-bar. Other variables, e.g. transition matrices $A_{j}$, are discernible by the inter-period step subscript $j$.

An LTI system is a trivial instance of a linear periodic system, with period length $p=1$. Non-trivial periodic systems with $p \geq 2$ arise naturally in a number of situations $[13,22]$. In this paper they are employed as models of linear systems with asynchronous control inputs. Specifically multirate and multiplexed control inputs are considered.

\section{A. Input-Multirate Systems}

Consider an LTI system of Definition 1. Each dimension $u_{[c]} \forall c \in \mathbb{N}_{1}^{m}$ of control input $u$ is called an input channel. In usual discrete-time control formulations each input channel is updated simultaneously. In the input-multirate control framework each input channel $u_{[c]}$ may have a unique sampleperiod $p_{c} \in \mathbb{N}_{+}$associated with it. Consider the timing diagram of Fig. 1. From top to bottom, three input channels and system step index $i$ are depicted. Input channel $u_{[1]}$ has a sample-period of three; $p_{1}=3$. Input channel $u_{[2]}$ has a sample-period of two; $p_{2}=2$. Input channel $u_{[1]}$ is updated at every step $i ; p_{3}=1$. Input $u_{[c] i}$ can be updated only if $\bmod \left(i, p_{c}\right)=0$. If $\bmod \left(i, p_{c}\right) \neq 0$ then $u_{[c] i}=u_{[c] i-1}$.

Employing this framework the entire control input vector $u$, i.e. each input channel, is initialized at system step $i=0$. Let $p:=\min \left\{l \in \mathbb{N}_{+} \mid \bmod \left(l, p_{c}\right)=0 \forall c \in \mathbb{N}_{1}^{m}\right\} \leq \prod_{c=1}^{m} p_{c}$ and note that the entire control input vector is updated at integer multiples of $p(i=0, p, 2 p, \ldots)$. Consider again Fig. 1. In this case $p=6$. Steps when the entire control input vector $u$ is updated are marked with a dashed vertical line. System (1) subject to constraints (2) and with multiple input-rates can be expressed as linear periodic system (3) subject to constraints (4) as explained next. Throughout the following explanation it holds that inter-period step index $j=\bmod (i, p)$.

For every $j \in \mathbb{N}_{0}^{p-1}$ first determine the number of channels $m_{j} \leq m$ which can be updated at step $j$, i.e. all decision variables at step $j$. Let $n_{j}:=n+m-m_{j} \forall j \in \mathbb{N}_{0}^{p-1}$. Then define $\left[\mathcal{C}_{j} \in\{0,1\}^{m \times m_{j}}\right] \wedge\left[\mathcal{D}_{j} \in\{0,1\}^{m \times\left(m-m_{j}\right)}\right]$ $\forall j \in \mathbb{N}_{0}^{p-1}$ such that control input $u_{i}$ is split according to

$$
u_{i}=\mathcal{C}_{j} \bar{u}_{i}+\mathcal{D}_{j} \hat{u}_{i}
$$

where periodic control input $\bar{u}_{i} \in \mathbb{R}^{m_{j}}$ contains all input channels which are updated at step $i$, and $\hat{u}_{i} \in \mathbb{R}^{m-m_{j}}$ contains all input channels which remain unchanged from the previous step $i-1$. Next define periodic state $\bar{x}_{i}:=$ $\left[x_{i}^{\mathrm{T}}, \hat{u}_{i}^{\mathrm{T}}\right]^{\mathrm{T}} \in \mathbb{R}^{n_{j}}$ and matrices $\mathcal{E}_{j}:=\left[I_{n}, 0_{\left\{n, m-m_{j}\right\}}\right] \wedge$

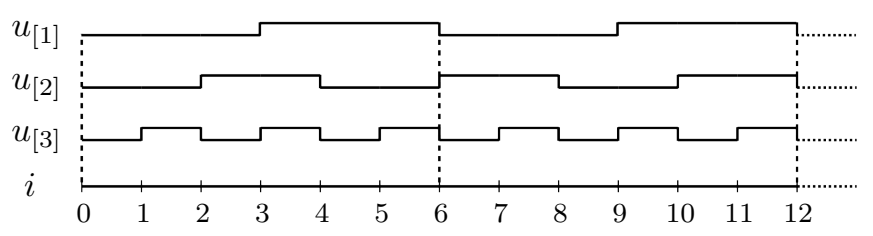

Fig. 1. Input-multirate control timing: $\left(p_{1}, p_{2}, p_{3}\right)=(3,2,1), p=6$. 


$$
\begin{gathered}
\mathcal{F}_{j}:=\left[0_{\left\{m-m_{j}, n\right\}}, I_{m-m_{j}}\right] \forall j \in \mathbb{N}_{0}^{p-1} \text { such that } \\
x_{i}=\mathcal{E}_{j} \bar{x}_{i} \wedge \hat{u}_{i}=\mathcal{F}_{j} \bar{x}_{i} .
\end{gathered}
$$

Then define matrices $\left[\mathcal{A}_{j} \in \mathbb{R}^{n_{\bmod (j+1, p)} \times n}\right] \wedge\left[\mathcal{B}_{j} \in\right.$ $\left.\mathbb{R}^{n_{\bmod (j+1, p)} \times m}\right] \forall j \in \mathbb{N}_{0}^{p-1}$ such that

$$
\bar{x}_{i+1}=\mathcal{A}_{j} x_{i}+\mathcal{B}_{j} u_{i} .
$$

Combining Eqs. (2), (5), (6) and (7) achieves dynamics

$$
\begin{aligned}
\bar{x}_{i+1} & =\mathcal{A}_{j} \mathcal{E}_{j} \bar{x}_{i}+\mathcal{B}_{j}\left(\mathcal{C}_{j} \bar{u}_{i}+\mathcal{D}_{j} \mathcal{F}_{j} \bar{x}_{i}\right) \\
& =\left(\mathcal{A}_{j} \mathcal{E}_{j}+\mathcal{B}_{j} \mathcal{D}_{j} \mathcal{F}_{j}\right) \bar{x}_{i}+\mathcal{B}_{j} \mathcal{C}_{j} \bar{u}_{i}
\end{aligned}
$$

subject to constraints

$$
\begin{aligned}
& E \mathcal{E}_{j} \bar{x}_{i}+G\left(\mathcal{C}_{j} \bar{u}_{i}+\mathcal{D}_{j} \mathcal{F}_{j} \bar{x}_{i}\right)= \\
&\left(E \mathcal{E}_{j}+G \mathcal{D}_{j} \mathcal{F}_{j}\right) \bar{x}_{i}+G \mathcal{C}_{j} \bar{u}_{i} \leq W .
\end{aligned}
$$

For all $j \in \mathbb{N}_{0}^{p-1}$, defining $A_{j}:=\mathcal{A}_{j} \mathcal{E}_{j}+\mathcal{B}_{j} \mathcal{D}_{j} \mathcal{F}_{j}, B_{j}:=$ $\mathcal{B}_{j} \mathcal{C}_{j}, E_{j}:=E \mathcal{E}_{j}+G \mathcal{D}_{j} \mathcal{F}_{j}, G_{j}:=G \mathcal{C}_{j}$ and $W_{j}=W$ results in system (3) subject to constraints (4).

It holds that $\left[n_{j} \geq n\right] \wedge\left[m_{j} \leq m\right] \wedge\left[n_{j}+m_{j}=n+m\right]$ $\forall j \in \mathbb{N}_{0}^{p-1}$. This means that the total amount of information required to describe the system $\left(n_{j}\right)$ changes depending on the number of 'saved' control inputs $\left(m-m_{j}\right)$. However, the number of saved control inputs and the number of inputs which are decision variables $\left(m_{j}\right)$ together must remain constant and equal to $m$. Furthermore $\left[n_{0}=n\right] \wedge\left[m_{0}=m\right]$. This is because at time $i=0$ and multiples of period-length $p$ every input channel of control vector $u$ can be updated.

To illustrate, the example of Fig. 1 results in the following, where $m=3$ as drawn, and all 0 's and 1's are scalar:

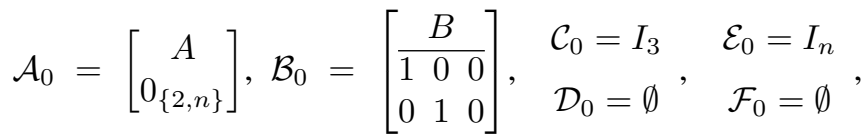

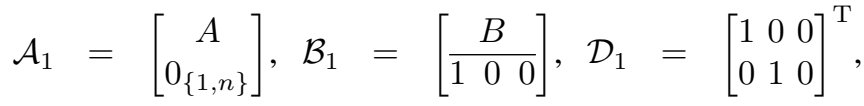

$$
\begin{aligned}
& \mathcal{C}_{1}=\left[\begin{array}{lll}
0 & 0 & 1
\end{array}\right]^{\mathrm{T}}, \mathcal{E}_{1}=\left[I_{n}, 0_{\{n, 2\}}\right], \mathcal{F}_{1}=\left[0_{\{2, n\}}, I_{2}\right] .
\end{aligned}
$$

Note that $\mathcal{D}_{0}=\emptyset$ and $\mathcal{F}_{0}=\emptyset$ because $\hat{u}$ has dimension 0 , i.e. at step $j=0$ every input channel is a decision variable.

\section{B. Input-Multiplexed Systems}

Consider continuous-time linear time-invariant system

$$
\dot{x}(t)=A_{\mathrm{c}} x(t)+B_{\mathrm{c}} u(t)
$$

on time $t \in \mathbb{R}$ with state $x \in \mathbb{R}^{n}, n \in \mathbb{N}_{+}$, control input $u \in \mathbb{R}^{m}, m \in \mathbb{N}_{+}, A_{\mathrm{c}} \in \mathbb{R}^{n \times n}$ and $B_{\mathrm{c}} \in \mathbb{R}^{n \times m}$.

In the multiplexed control framework the continuous-time control input is piecewise constant on each input channel, but

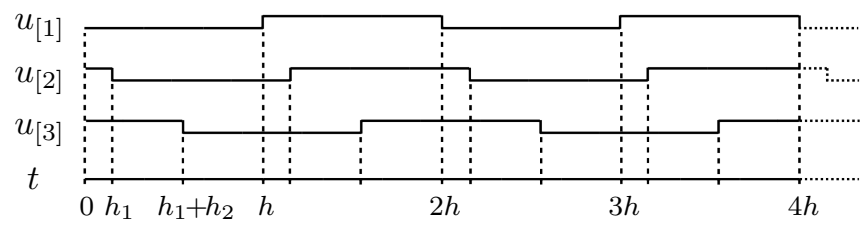

Fig. 2. Multiplexed control timing: $m=p=3, h=h_{1}+h_{2}+h_{3}$. all input channels are not updated simultaneously. However, each input channel is updated at the same, fixed time-interval $h \in \mathbb{R}_{+}$. Fig. 2 shows a timing schematic of such a system. Without loss of generality suppose the input channels are numbered such that channel $u_{[\bmod (c, m)+1]}$ is updated next after channel $u_{[c]}$. Associated with each input channel $u_{[c]}$ is a fixed time interval $h_{c}$ satisfying: $h_{c} \in \mathbb{R}_{0} \forall c \in \mathbb{N}_{1}^{m-1}$, $h_{m} \in \mathbb{R}_{+}$. Each $h_{c}$ denotes the time interval between input channels $u_{[c]}$ and $u_{[\bmod (c, m)+1]}$ being updated. Note that $h=$ $\sum_{c=1}^{m} h_{c}$, and that if $h_{c}=0$ for some $c \in \mathbb{N}_{1}^{m-1}$ then input channels $u_{[c]}$ and $u_{[c+1]}$ are updated simultaneously.

For control law synthesis it may be desirable to update each input channel individually $[12,17]$. Thus suppose $h_{c}>$ $0 \forall c \in \mathbb{N}_{1}^{m}$. Continuous-time system (8) with piecewise constant control inputs can be rewritten as linear periodic system (3) with period length $p=m$ and $\left[A_{j}:=\exp \left(A_{\mathrm{c}} h_{j}\right) \in\right.$ $\left.\mathbb{R}^{n \times n}\right] \wedge\left[B_{j}:=\int_{0}^{h_{j}} \exp \left(A_{\mathrm{c}} \tau\right) \mathrm{d} \tau B_{\mathrm{c}} \in \mathbb{R}^{n \times m}\right] \forall j \in \mathbb{N}_{0}^{p-1}$. The multiplexed inputs, and pointwise-in-time constraints of the form of Eq. (2), are incorporated by following an analogous sequence of steps as described in Sec. II-A for input-multirate LTI systems. The system with multiplexed input channels is then expressed as linear periodic system (3) with $\left[n_{j}=n+m-1\right] \wedge\left[m_{j}=1\right] \forall j \in \mathbb{N}_{0}^{p-1}$. This means there is only one decision variable at each step $i$. All other input channel values are 'saved' in periodic state $\bar{x}$.

Note that if some $h_{c}$ 's are allowed to be zero then the state and input dimension may be time-dependent.

\section{MPC OF LINEAR PERIODIC SySTEMS}

Control law synthesis and the design of MPC problems is tackled in this section. The framework of state-feedback is employed assuming perfect state information. Regulation to the origin is considered. The methods are applicable to offsetfree or trajectory tracking (see Sec. V). The methods could straightforwardly be bestowed with robustness properties. For simplicity of exposition robustness is ignored here.

\section{A. A Basic MPC Problem Formulation}

For prediction horizon length $N \in \mathbb{N}_{+}$, MPC achieves closed-loop control action of system (3) subject to constraints (4) by applying at each step $i$ the first control input $\bar{u}_{(i, 0)}$ of a predicted open-loop control input trajectory $\bar{U}_{i}:=\left[\bar{u}_{(i, 0)}^{\mathrm{T}}\right.$, $\left.\ldots, \bar{u}_{(i, N-1)}^{\mathrm{T}}\right]^{\mathrm{T}} \in \mathbb{R}^{\nu_{j}}$, where $\bar{u}_{(i, k)}$ denotes the future value of $\bar{u}$ at step $i+k$, as predicted from step $i$. The dimension $\nu_{j}$ of $\bar{U}_{i}$ is not defined explicitly here, but depends on $i$, $N$ and $m_{j}$ 's, and is periodic. The optimal predicted openloop control input trajectory is determined by the solution of periodic linear-quadratic MPC Problem 1 with quadratic cost matrices $\left[T_{j} \in \mathbb{R}^{n_{j} \times n_{j}}\right] \wedge\left[\Gamma_{j} \in \mathbb{R}^{\left(n_{j}+m_{j}\right) \times\left(n_{j}+m_{j}\right)}\right] \forall j \in$ $\mathbb{N}_{0}^{p-1}$ and terminal constraint sets $\mathbb{T}_{j} \in 2^{\mathbb{R}^{n_{j}}} \forall j \in \mathbb{N}_{0}^{p-1}$. The parameters $N, T_{j}, \Gamma_{j}$ and $\mathbb{T}_{j}$ are design parameters. Specific values are assigned to terminal cost matrices $T_{j}$ and terminal constraint sets $\mathbb{T}_{j}$ in later sections to yield control laws with desirable properties. For compact notation define the extended periodic state $\bar{\chi}_{i}:=\left[\bar{x}_{i}^{\mathrm{T}}, \bar{u}_{i}^{\mathrm{T}}\right]^{\mathrm{T}}$ and $\bar{A}_{j}:=\left[A_{j}, B_{j}\right]$ $\wedge \bar{E}_{j}:=\left[E_{j}, G_{j}\right] \forall j \in \mathbb{N}_{0}^{p-1}$. 
Problem 1: Determine

$$
\bar{U}_{i}^{*}\left(\bar{x}_{i}\right):=\arg \min _{\bar{U}_{i} \in \mathcal{U}\left(\bar{x}_{i}\right)} J\left(\bar{x}_{i}, \bar{U}_{i}\right)
$$

with prediction cost function $J: \mathbb{R}^{n_{j}} \times \mathbb{R}^{\nu_{j}} \rightarrow \mathbb{R}$

$$
\begin{aligned}
J\left(\bar{x}_{i}, \bar{U}_{i}\right):=\quad \sum_{k=0}^{N-1} \bar{\chi}_{(i, k)}^{\mathrm{T}} \Gamma_{\bmod (i+k, p)} \bar{\chi}_{(i, k)} \\
+\bar{x}_{(i, N)}^{\mathrm{T}} T_{\bmod (i+N, p)} \bar{x}_{(i, N)}
\end{aligned}
$$

constraint set $\mathcal{U}: \mathbb{R}^{n_{j}} \rightarrow\left\{2^{\mathbb{R}^{\nu_{j}}}, \emptyset\right\}$,

$\mathcal{U}\left(\bar{x}_{i}\right):=\left\{\begin{array}{l|l}\bar{U}_{i} \in \mathbb{R}^{\nu_{j}} & \begin{array}{l}\bar{x}_{(i, N)} \in \mathbb{T}_{\bmod (i+N, p)} \\ \bar{E}_{\bmod (i+k, p)} \bar{\chi}_{(i, k)} \leq W_{\bmod (i+k, p)} \\ \forall k \in \mathbb{N}_{0}^{N-1}\end{array}\end{array}\right\}$,

and $\bar{x}_{(i, k+1)}=\bar{A}_{\bmod (i+k, p)} \bar{\chi}_{(i, k)} \forall k \in \mathbb{N}_{0}^{N-1}$.

Periodic MPC Problem 1 is essentially identical to a usual time-invariant MPC problem. There are stage costs, terminal costs, stage constraints and a terminal constraint. The crucial difference is their periodic nature, and that depending on $i$ and $N$ the terminal cost and constraint are different.

Define the sets $\mathbb{X}_{j} \in 2^{\mathbb{R}^{n_{j}}} \forall j \in \mathbb{N}_{0}^{p-1}$ of feasible states as follows: $\mathbb{X}_{j}:=\left\{\bar{x} \in \mathbb{R}^{n_{j}} \mid \mathcal{U}(\bar{x}) \neq \emptyset\right\}$.

Definition 3: An MPC problem is strongly feasible if and only if from every feasible state the closed-loop state trajectory due to any sequence of feasible solutions remains within the sets of feasible states.

Definition 4: Suppose a specific set of parameters $\left\{N, T_{j}\right.$, $\left.\Gamma_{j}, \mathbb{T}_{j}\right\}$ in MPC Problem 1 results in the sets of feasible states $\hat{\mathbb{X}}_{j} \in 2^{\mathbb{R}^{n_{j}}} \forall j \in \mathbb{N}_{0}^{p-1}$. Then MPC Problem 1 with $\left\{N, T_{j}, \Gamma_{j}, \mathbb{T}_{j}\right\}$ is termed least-restrictive if and only if any other choice of parameters results in $\mathbb{X}_{j} \subseteq \hat{\mathbb{X}}_{j} \forall j \in \mathbb{N}_{0}^{p-1}$.

In MPC of LTI systems, strong feasibility of MPC problems can be enforced by constraining terminal state $x_{(i, N)}$ to be element of a controlled invariant set. Constraining the terminal state to the maximum controlled invariant set results in a least-restrictive strongly feasible MPC problem. Stability can be enforced by selecting an appropriate terminal cost in combination with a more restrictive terminal constraint. For example, the terminal cost matrix may be the stabilizing, positive-definite solution $P$ to a discrete-time algebraic Riccati equation, and the terminal constraint set a positively invariant set for the optimal state-feedback associated with $P$. If the terminal constraint is then not active for some particular state, i.e. the terminal state is an element of the positively invariant set without this being explicitly enforced, then the solution of the MPC problem is infinite-horizon optimal. These issues are thoroughly discussed in [15].

The point of this paper it to propose that these basic ingredients for enforcing strong feasibility and stability, and for verifying optimality, are no different in a periodic MPC problem formulation. The concept of (maximum) controlled and positively invariant sets, and Riccati equations, are identical in the linear periodic case. The differences associated with the system's periodicity are numerical, rather than conceptual. In Sections III-B and III-C a definition of periodic (maximum) controlled and positively invariant sets is proposed, and a solution method presented. Sec. III-D presents a short discussion on the solution of reverse periodic discrete-time algebraic Riccati equations. The objective is to derive an analogous set of results for guaranteeing strong feasibility, stability and optimality as for MPC of LTI systems, but generalized to the linear periodic systems framework.

B. Controlled Invariance of Linear Periodic Systems and Enforcing Strong Feasibility of Periodic MPC Problems

The purpose of this section is to formally characterize controlled invariance [3] of linear periodic systems, and employ maximum periodic controlled invariant sets in the design of least-restrictive strongly feasible periodic MPC problems. Furthermore, an approach for the computation of maximum periodic controlled invariant sets is presented.

Definition 5: A set $\left\{\mathbb{C}_{0}, \ldots, \mathbb{C}_{p-1}\right\}$ of sets $\mathbb{C}_{j} \in 2^{\mathbb{R}^{n_{j}}} \forall j \in$ $\mathbb{N}_{0}^{p-1}$ is termed a periodic controlled invariant set for system (3) subject to (4) iff it satisfies the following condition:

$$
\begin{gathered}
\forall j \in \mathbb{N}_{0}^{p-1} \forall \bar{x} \in \mathbb{C}_{j} \quad \exists \bar{u} \in \mathbb{R}^{m_{j}} \quad \text { s.t. } \\
A_{j} \bar{x}+B_{j} \bar{u} \in \mathbb{C}_{\bmod (j+1, p)} \quad \wedge \quad E_{j} \bar{x}+G_{j} \bar{u} \leq W_{j} .
\end{gathered}
$$

The maximum periodic controlled invariant set is the set $\left\{\mathbb{C}_{0}^{*}, \ldots, \mathbb{C}_{p-1}^{*}\right\}$ of sets $\mathbb{C}_{j}^{*} \in 2^{\mathbb{R}^{n_{j}}} \forall j \in \mathbb{N}_{0}^{p-1}$ as follows:

$$
\begin{aligned}
\mathbb{C}_{j}^{*}:= & \left\{\bar{x} \in \mathbb{R}^{n_{j}} \mid \exists\left\{\bar{u}_{i} \in \mathbb{R}^{\left.m_{\bmod (i, p)}\right\}_{i=j}^{\infty} \text { s.t. }}\right.\right. \\
& \bar{x}_{i+1}=A_{k} \bar{x}_{i}+B_{k} \bar{u}_{i} \wedge E_{k} \bar{x}_{i}+G_{k} \bar{u}_{i} \leq W_{k} \\
& \left.\wedge k=\bmod (i, p) \quad \forall i \in \mathbb{N}_{j}^{\infty}, \bar{x}_{j}=\bar{x}\right\} .
\end{aligned}
$$

Theorem 1: Setting terminal constraint sets $\mathbb{T}_{j}=\mathbb{C}_{j}^{*} \forall j \in$ $\mathbb{N}_{0}^{p-1}$ in MPC Problem 1 results in a least-restrictive strongly feasible MPC problem, for any $N \in \mathbb{N}_{+}$.

Proof: A formal proof is omitted for brevity. This outline follows closely the discussion of [15] for time-invariant systems. Strong feasibility is enforced because if at feasible state $\bar{x}_{i}$ MPC Problem 1 determines any solution $\bar{U}_{i}$ such that $\bar{x}_{(i, N)} \in \mathbb{C}_{\bmod (i+N, p)}^{*}$ then at the next state $\bar{x}_{i+1}=\bar{x}_{(i, 1)}$ an admissible control input trajectory up to but not including the final control move $\bar{u}_{(i+1, N-1)}$ is given by the shifted solution of the step before: $\bar{u}_{(i+1, k)}=\bar{u}_{(i, k+1)} \forall k \in \mathbb{N}_{0}^{N-2}$. Applying this achieves $\bar{x}_{(i+1, N-1)} \in \mathbb{C}_{\bmod (i+N, p)}^{*}$. The existence of an admissible final control move $\bar{u}_{(i+1, N-1)}$ such that $\bar{x}_{(i+1, N)} \in \mathbb{C}_{\bmod (i+N+1, p)}^{*}$ is guaranteed by the periodic controlled invariance property of Eq. (9). By induction this argument holds recursively. Least-restrictiveness of the MPC problem results from the terminal constraint set being the maximum periodic controlled invariant set.

Any periodic controlled invariant set can be employed for enforcing strong feasibility of MPC Problem 1. Use of a non-maximum periodic controlled invariant set may result in a restrictive control law. Note that a strongly feasible MPC problem does not necessarily yield a stabilizing control law.

Define the lifted system matrices

$$
\begin{aligned}
\mathscr{A} & :=\left[\begin{array}{cc}
0 & A_{p-1} \\
\operatorname{diag}\left(A_{0}, \ldots, A_{p-2}\right) & 0
\end{array}\right] \in \mathbb{R}^{\bar{n} \times \bar{n}}, \\
\mathscr{B} & :=\left[\begin{array}{cc}
0 & B_{p-1} \\
\operatorname{diag}\left(B_{0}, \ldots, B_{p-2}\right) & 0
\end{array}\right] \in \mathbb{R}^{\bar{n} \times \bar{m},}
\end{aligned}
$$




$$
\begin{aligned}
\mathscr{E} & :=\operatorname{diag}\left(E_{0}, \ldots, E_{p-1}\right) \in \mathbb{R}^{\bar{r} \times \bar{n}}, \\
\mathscr{G} & :=\operatorname{diag}\left(G_{0}, \ldots, G_{p-1}\right) \in \mathbb{R}^{\bar{r} \times \bar{m}}, \\
\mathscr{W} & :=\left[W_{0}^{\mathrm{T}}, \ldots, W_{p-1}^{\mathrm{T}}\right]^{\mathrm{T}} \in \mathbb{R}^{\bar{r}}, \\
\bar{n} & :=\sum_{j=0}^{p-1} n_{j}, \quad \bar{m}:=\sum_{j=0}^{p-1} m_{j}, \quad \bar{r}:=\sum_{j=0}^{p-1} r_{j},
\end{aligned}
$$

and consider the lifted LTI system

$$
x_{i+1}=\mathscr{A} x_{i}+\mathscr{B} u_{i} \text { subject to } \mathscr{E} x_{i}+\mathscr{G} u_{i} \leq \mathscr{W} .
$$

An equivalent condition to Eq. (9) is the following:

$$
\begin{array}{r}
\forall x \in \mathbb{C}:=\left(\mathbb{C}_{0} \times \cdots \times \mathbb{C}_{p-1}\right) \in 2^{\mathbb{R}^{\bar{n}}} \exists u \in \mathbb{R}^{\bar{m}} \\
\text { s.t. } \mathscr{A} x+\mathscr{B} u \in \mathbb{C} \wedge \mathscr{E} x+\mathscr{G} u \leq \mathscr{W} .
\end{array}
$$

Given the maximum controlled invariant set $\mathbb{C}^{*} \in 2^{\mathbb{R}^{\bar{n}}}$ of lifted LTI system (10) according to (see [3])

$$
\begin{aligned}
\mathbb{C}^{*}:=\left\{x_{0} \in \mathbb{R}^{\bar{n}} \mid \exists\left\{u_{i} \in \mathbb{R}^{\bar{m}}\right\}_{i=0}^{\infty}\right. \text { s.t. } \\
\left.x_{i+1}=\mathscr{A} x_{i}+\mathscr{B} u_{i} \wedge \mathscr{E} x_{i}+\mathscr{G} u_{i} \leq \mathscr{W} \quad \forall i \in \mathbb{N}\right\}
\end{aligned}
$$

it is straightforward to unlift the set $\mathbb{C}^{*}$ and determine the maximum periodic controlled invariant set $\left\{\mathbb{C}_{0}^{*}, \ldots, \mathbb{C}_{p-1}^{*}\right\}$ such that $\mathbb{C}^{*}=\left(\mathbb{C}_{0}^{*} \times \cdots \times \mathbb{C}_{p-1}^{*}\right)$. Thus the determination of maximum periodic controlled invariant sets is amenable to well-known methods from LTI system theory $[3,7,23]$.

\section{Positive Invariance of Linear Periodic Systems}

The purpose of this section is to formally characterize positive invariance [3] of linear periodic systems. Furthermore, an approach for the computation of maximum periodic positively invariant sets is presented.

Definition 6: A set $\left\{\mathbb{P}_{0}, \ldots, \mathbb{P}_{p-1}\right\}$ of sets $\mathbb{P}_{j} \in 2^{\mathbb{R}^{n_{j}}} \forall j \in$ $\mathbb{N}_{0}^{p-1}$ is termed a periodic positively invariant set for system (3) subject to (4) with state-feedback gains $\left\{K_{0}, \ldots, K_{p-1}\right\}$, $K_{j} \in \mathbb{R}^{m_{j} \times n_{j}}$ iff it satisfies the following condition:

$$
\begin{aligned}
&\left(A_{j}+B_{j} K_{j}\right) \bar{x} \in \mathbb{P}_{\bmod (j+1, p)} \wedge \\
&\left(E_{j}+G_{j} K_{j}\right) \bar{x} \leq W_{j} \quad \forall \bar{x} \in \mathbb{P}_{j} \quad \forall j \in \mathbb{N}_{0}^{p-1} .
\end{aligned}
$$

The maximum periodic positively invariant set is the set $\left\{\mathbb{P}_{0}^{*}, \ldots, \mathbb{P}_{p-1}^{*}\right\}$ of sets $\mathbb{P}_{j}^{*} \in 2^{\mathbb{R}^{n_{j}}} \forall j \in \mathbb{N}_{0}^{p-1}$ as follows:

$$
\begin{aligned}
\mathbb{P}_{j}^{*}:= & \left\{\bar{x} \in \mathbb{R}^{n_{j}} \mid \bar{x}_{i+1}=\left(A_{k}+B_{k} K_{k}\right) \bar{x}_{i} \wedge\right. \\
& \left(E_{k}+G_{k} K_{k}\right) \bar{x}_{i} \leq W_{k} \wedge \\
& \left.k=\bmod (i, p) \quad \forall i \in \mathbb{N}_{j}^{\infty}, \quad \bar{x}_{j}=\bar{x}\right\} .
\end{aligned}
$$

Let $\mathscr{K}:=\operatorname{diag}\left(K_{0}, \ldots, K_{p-1}\right) \in \mathbb{R}^{\bar{m} \times \bar{n}}$. An equivalent condition to Eq. (13) is the following:

$$
\begin{aligned}
(\mathscr{A}+\mathscr{B} \mathscr{K}) x \in \mathbb{P}:= & \left(\mathbb{P}_{0} \times \cdots \times \mathbb{P}_{p-1}\right) \in 2^{\mathbb{R}^{\bar{n}}} \\
& \wedge(\mathscr{E}+\mathscr{G} \mathscr{K}) x \leq \mathscr{W} \quad \forall x \in \mathbb{P} .
\end{aligned}
$$

Given the maximum positively invariant set $\mathbb{P}^{*} \in 2^{\mathbb{R}^{\bar{n}}}$ of lifted LTI system (10) according to (see [3])

$$
\begin{aligned}
\mathbb{P}^{*}:=\left\{x_{0} \in \mathbb{R}^{\bar{n}} \mid x_{i+1}=\right. & (\mathscr{A}+\mathscr{B} \mathscr{K}) x_{i} \wedge \\
& \left.(\mathscr{E}+\mathscr{G} \mathscr{K}) x_{i} \leq \mathscr{W} \quad \forall i \in \mathbb{N}\right\}
\end{aligned}
$$

it is straightforward to unlift the set $\mathbb{P}^{*}$ and determine the maximum periodic positively invariant set $\left\{\mathbb{P}_{0}^{*}, \ldots, \mathbb{P}_{p-1}^{*}\right\}$ such that $\mathbb{P}^{*}=\left(\mathbb{P}_{0}^{*} \times \cdots \times \mathbb{P}_{p-1}^{*}\right)$. Thus the determination of maximum periodic positively invariant sets is amenable to well-known methods from LTI system theory $[3,8]$.
D. Reverse Periodic Discrete-Time Algebraic Riccati Equations and Guaranteeing Stability and Optimality of Periodic MPC Control Laws

The purpose of this section is to briefly summarize known results on the solution of reverse periodic discrete-time algebraic Riccati equations. Together with the periodic positively invariant sets of Sec. III-C these are employed in the design of periodic MPC problems which result in stabilizing and infinite-horizon optimal control laws.

Consider a linear periodic system of Definition 2, and the unconstrained periodic linear-quadratic optimal control problem of minimizing the cost

$J\left(\bar{x}_{i}\right):=\sum_{k=0}^{\infty} \bar{\chi}_{(i, k)}^{\mathrm{T}} \Gamma_{j} \bar{\chi}_{(i, k)} \quad, \quad \Gamma_{j}=\left[\begin{array}{cc}Q_{j} & S_{j} \\ S_{j}^{\mathrm{T}} & R_{j}\end{array}\right] \geq 0$

with $j=\bmod (i+k, p)$ and $\bar{\chi}$ defined as in MPC Problem 1. The optimal cost $J^{*}$ is given by

$$
J^{*}\left(\bar{x}_{i}\right)=\bar{x}_{i}^{\mathrm{T}} P_{j} \bar{x}_{i}
$$

and is achieved by periodic state-feedback

$$
\begin{aligned}
\bar{u}_{(i, k)} & =K_{j} \bar{x}_{(i, k)}, \\
K_{j} & :=-\left[B_{j}^{\mathrm{T}} P_{j+1} B_{j}+R_{j}\right]^{-1}\left(B_{j}^{\mathrm{T}} P_{j+1} A_{j}+S_{j}^{\mathrm{T}}\right) \\
& \in \mathbb{R}^{m_{j} \times n_{j}} \quad \forall j \in \mathbb{N}_{0}^{p-1}
\end{aligned}
$$

where $\left\{P_{0}, \ldots, P_{p-1}\right\}, P_{j} \in \mathbb{R}^{n_{j} \times n_{j}} \forall j \in \mathbb{N}_{0}^{p-1}$ is a unique, positive semi-definite periodically stabilizing solution of the reverse periodic discrete-time algebraic Riccati equation

$$
\begin{aligned}
P_{j}= & Q_{j}+A_{j}^{\mathrm{T}} P_{j+1} A_{j}-\left(B_{j}^{\mathrm{T}} P_{j+1} A_{j}+S_{j}^{\mathrm{T}}\right)^{\mathrm{T}} \\
& \times\left[B_{j}^{\mathrm{T}} P_{j+1} B_{j}+R_{j}\right]^{-1}\left(B_{j}^{\mathrm{T}} P_{j+1} A_{j}+S_{j}^{\mathrm{T}}\right) .
\end{aligned}
$$

The important point about these periodic Riccati equations is that each $P$ matrix may have a different dimension. Conditions for the existence of solutions are discussed in [5]. Solution methods for such equations are discussed in [5,19,21]. For usefulness in MPC we make Assumption 1. A less strict condition than Assumption 1 is possible, but for simplicity of exposition this is ignored here.

Assumption 1: Stage cost matrices are positive definite: $\Gamma_{j} \succ 0 \forall j \in \mathbb{N}_{0}^{p-1}$.

Suppose the solution $\left\{P_{0}, \ldots, P_{p-1}\right\}$, the set of feedback gains $\left\{K_{0}, \ldots, K_{p-1}\right\}$, and any appropriate positively invariant set $\left\{\mathbb{P}_{0}, \ldots, \mathbb{P}_{p-1}\right\}$ have been determined.

Theorem 2: Setting terminal costs $T_{j}=P_{j}$ and terminal constraint $\mathbb{T}_{j}=\mathbb{P}_{j} \forall j \in \mathbb{N}_{0}^{p-1}$ in MPC Problem 1 results in a stabilizing control law.

Proof: The proof follows closely along the lines of the discussion of [15] for LTI systems. Basically, the minimal prediction cost $J\left(\bar{x}_{i}, \bar{U}_{i}^{*}\right)$ is a strict upper bound for the minimal prediction cost $J\left(\bar{x}_{i+1}, \bar{U}_{i+1}^{*}\right)$ at the next step. Thus the minimal prediction cost is guaranteed to be monotonically decreasing and is thus a Lyapunov function for the closed-loop system. To see this consider a feasible state $\bar{x}_{i}$. 
The optimal solution $\bar{U}_{i}^{*}$ achieves $\bar{x}_{(i, N)} \in \mathbb{P}_{\bmod (i+N, p)}$. Analogously to the proof of Theorem 1, at the next state $\bar{x}_{i+1}=\bar{x}_{(i, 1)}$ a feasible solution $\bar{U}_{i+1}$ is such that

$$
\begin{aligned}
\bar{u}_{(i+1, k)} & =\bar{u}_{(i, k+1)} \quad \forall k \in \mathbb{N}_{0}^{N-2} \\
\bar{u}_{(i+1, N-1)} & =K_{\bmod (i+N, p)} \bar{x}_{(i+1, N-1)} .
\end{aligned}
$$

Therefore

$$
J\left(\bar{x}_{i+1}, \bar{U}_{i+1}^{*}\right)-J\left(\bar{x}_{i}, \bar{U}_{i}^{*}\right) \leq-\bar{\chi}_{i}^{\mathrm{T}} \Gamma_{\bmod (i, p)} \bar{\chi}_{i}<0
$$

by Assumption 1 .

Theorem 3: Suppose $T_{j}=P_{j}$ and $\mathbb{T}_{j}=\mathbb{P}_{j} \forall j \in \mathbb{N}_{0}^{p-1}$ in MPC Problem 1. If at state $\bar{x}_{i}$ the optimal solution $\bar{U}_{i}^{*}$ is such that the terminal constraint is not active, then the solution is the infinite-horizon optimal solution.

Proof: The proof again follows along the lines of the discussion of [15], and the proof of Theorem 2. Basically, the optimal solution $U_{i}^{*}\left(\bar{x}_{i}\right)$ is the first portion of the actual control input trajectory that is determined in the closed-loop. As the terminal constraint is not active the terminal cost incorporates the exact cost associated with the infinite-horizon prediction beyond prediction horizon $N$. Consider a feasible state $\bar{x}_{i}$ and optimal solution $\bar{U}_{i}^{*}$ such that $\bar{x}_{(i, N)} \in \mathbb{P}_{\bmod (i+N, p)}$ with no active constraint on terminal state $\bar{x}_{(i, N)}$. Then at the next state $\bar{x}_{i+1}=\bar{x}_{(i, 1)}$ the optimal solution $\bar{U}_{i+1}^{*}$ is given by

$$
\begin{aligned}
\bar{u}_{(i+1, k)}^{*} & =\bar{u}_{(i, k+1)}^{*} \quad \forall k \in \mathbb{N}_{0}^{N-2} \\
\bar{u}_{(i+1, N-1)}^{*} & =K_{\bmod (i+N, p)} \bar{x}_{(i+1, N-1)} .
\end{aligned}
$$

\section{Consequently}

$$
J\left(\bar{x}_{i+1}, \bar{U}_{i+1}^{*}\right)-J\left(\bar{x}_{i}, \bar{U}_{i}^{*}\right)=-\bar{\chi}_{i}^{\mathrm{T}} \Gamma_{\bmod (i, p)} \bar{\chi}_{i}<0 .
$$

Thus the solution $U_{i}^{*}\left(\bar{x}_{i}\right)$ is both stabilizing and infinitehorizon optimal.

\section{Differences With Existing Methods}

In [18] an LTI system according to Definition 1 is first lifted to the LTI system

$$
\xi_{i+1}=\left[\begin{array}{cc}
A & B \\
0 & I_{m}
\end{array}\right] \xi_{i}+\left[\begin{array}{c}
B \\
I_{m}
\end{array}\right] \delta_{i}
$$

with $\xi_{i}:=\left[x_{i}^{\mathrm{T}}, u_{i}^{\mathrm{T}}\right]^{\mathrm{T}} \in \mathbb{R}^{n+m}$ and $\delta_{i}:=u_{i}-u_{i-1} \in \mathbb{R}^{m}$. Asynchronous control inputs are introduced by enforcing via the controller that $\delta_{[c] i}=0$ whenever input channel $u_{[c]}$ cannot be updated. This is akin to move-blocking. When using periodic models with time-dependent dimensions the input timing constraints are intrinsic to the plant, instead of being externally forced upon the system. This is instrumental in generalizing standard procedures for systems with synchronous inputs to the asynchronous setting. Control law design is significantly simplified and made more transparent. The complexities of handling time-dependent move-blocking data-structures to control input timing have been eradicated. Employing periodic models may also offer computational benefits. The lifted model of Eq. (16) has a combined stateinput dimension of $n+2 m$. The periodic model has only $n+m$. Thus fewer linear inequalities are required to describe the constraints on $(x, u)$ via $(\bar{x}, \bar{u})$, as opposed to via $(\xi, \delta)$. Note that the number of decision variables at each step $i$ is the same in both system representations. Note further that either system representation allows formulating MPC problems which result in infinite-horizon optimal control laws.

In $[12,17]$ the lifting strategy of Eq. (16) is employed, but a periodic input distribution matrix $B_{j}$ defined. The state transition matrix remains time-invariant. Again, using fully periodic models may reduce the complexity of the MPC problem, and of determining the required invariant sets.

In RMPC [11] the periodic system is lifted to an LTI system. The lifting is over an entire period. MPC is then performed as for LTI systems, but including inter-period constraints. Thus RMPC is agnostic to time-dependent dimensions and applicable to the linear periodic systems investigated here (see Sec. V). The drawback of RMPC is that the lifting to an LTI system reduces the effective sampling-rate of the controller to $1 / p$ that of the system. Thus the controller is not able to respond to disturbances and references changes on inter-period steps, but can only reject the effects of these on steps which are integer multiples of the period length. The periodic MPC approach of this paper rejects disturbances, and responds to reference changes, at each step. Furthermore, in periodic MPC the performance degradation due to disturbances is unrelated to the system's period length $p$, whereas a larger period length $p$ exacerbates the effects of the same levels of disturbances in RMPC. Furthermore, RMPC requires the prediction horizon to be an integer multiple of the period length. The periodic MPC approach proposed here has no such restriction. Thus the shortest admissible horizon length can be selected freely.

\section{ILlustrative EXAMPLE}

Consider the two-mass system shown in Fig. 3, which is a very simplified model of a common setup used in nanopositioning systems. In a standard configuration the larger mass $m_{b}$ would be, for example, a linear motor with a large travel, and the smaller mass $m_{p}$ a piezo element for high precision positioning and disturbance rejection, but small travel. The continuous-time dynamics are given by ( $n=4, m=2)$

$$
\begin{aligned}
& \ddot{x}_{b}=\frac{1}{m_{b}}\left[-k x_{b}-\left(\delta_{b}+\delta_{p}\right) \dot{x}_{b}+k x_{p}+\delta_{p} \dot{x}_{p}+f_{b}\right] \\
& \ddot{x}_{p}=\frac{1}{m_{p}}\left[k x_{b}+\delta_{p} \dot{x}_{b}-k x_{p}-\delta_{p} \dot{x}_{p}+f_{p}\right] .
\end{aligned}
$$

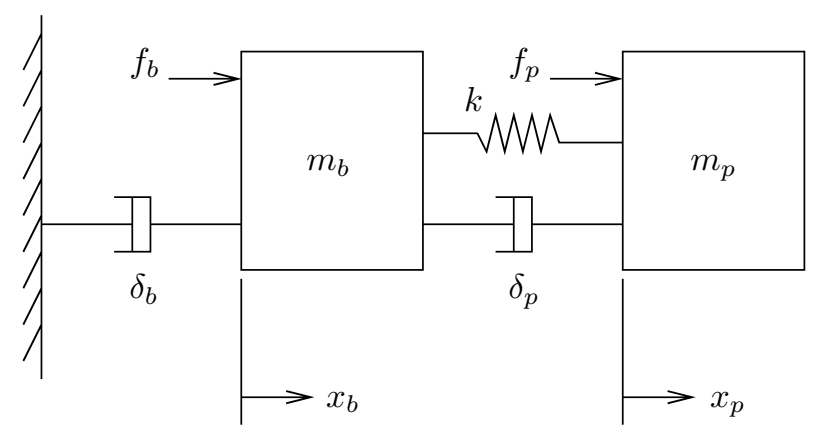

Fig. 3. Nano-positioning system schematic: Two-mass system. 
The two control inputs are the base- and piezo-stage forces $f_{b}$ and $f_{p}$. The mass, damping and spring coefficients are

$$
\begin{array}{rlrl}
m_{b}=10 \mathrm{~g} & \delta_{b} & =10^{-3} \mathrm{~N} \mathrm{~s} / \mathrm{m} \\
m_{p}=1 \mathrm{~g} & \delta_{p} & =5 \cdot 10^{-6} \mathrm{Ns} / \mathrm{m} \\
k & =0.25 \mathrm{~N} / \mathrm{m} .
\end{array}
$$

These parameters result in a very approximate model of the atomic force microscope (AFM) studied in [9], with a slow and heavy base-stage and a very lightly damped and fast piezo-stage. The system is sampled at $1 \mathrm{kHz}$. Due to the slow dynamics of the base, the input $f_{b}$ is updated only once every $p=10$ samples, resulting in a periodically time-dependent system with state and control input dimension alternating between 4 and 5 , and 1 and 2, respectively, as discussed in Sec. II-A. The following constraints are imposed:

$$
\begin{array}{rlrl}
\left|x_{b}\right| & \leq 0.4 \mathrm{~mm} & \\
\left|x_{p}\right| \leq 0.4 \mathrm{~mm} & \left|x_{b}-x_{p}\right| \leq 0.1 \mathrm{~mm} \\
\left|\dot{x}_{b}\right| \leq 0.03 \mathrm{~m} / \mathrm{s} & \left|\dot{x}_{b}-\dot{x}_{p}\right| \leq 0.1 \mathrm{~m} / \mathrm{s} \\
\left|f_{b}\right| \leq 5 \mathrm{mN} & \left|f_{p}\right| \leq 0.2 \mathrm{mN} .
\end{array}
$$

The control objective is to track a reference signal in the piezo-stage position $x_{p}$ as the AFM executes, for example, a raster scan. The reference trajectory switches between values of $\pm 0.4 \mathrm{~mm}$. As is the case in practice, the entire reference signal is known prior to the scan. This causes the system to respond, in a non-causal manner, to reference changes before they occur. Cost matrices $Q$ and $R$ equal to the identity, and a prediction horizon of $50 \mathrm{~ms}(N=50)$ were chosen. The choice of $N$ is sufficiently large such that the solution of the finite-horizon MPC problem is infinite-horizon optimal.

Plotted in Figs. 4 and 5 are the piezo-stage position $x_{p}$ and the base-stage velocity $\dot{x}_{b}$, respectively. Two control laws are contrasted; the optimal constrained periodic MPC control law proposed in this paper (blue, solid), and optimal unconstrained control based on periodic models and the solution of the periodic Riccati equation (red, dashed). The

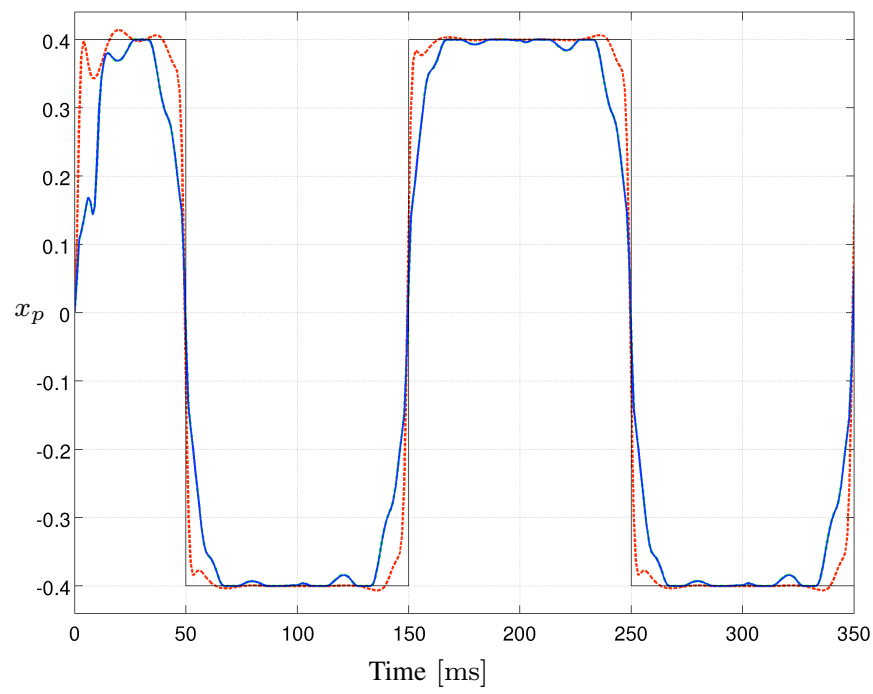

Fig. 4. Piezo-stage position $x_{p}[\mathrm{~mm}]$, undisturbed. Constrained optimal periodic MPC (blue, solid). Unconstrained optimal periodic control (red, dashed). Repetitive-MPC (green, dash-dotted). Note constraint $\left|x_{p}\right| \leq 0.4$.

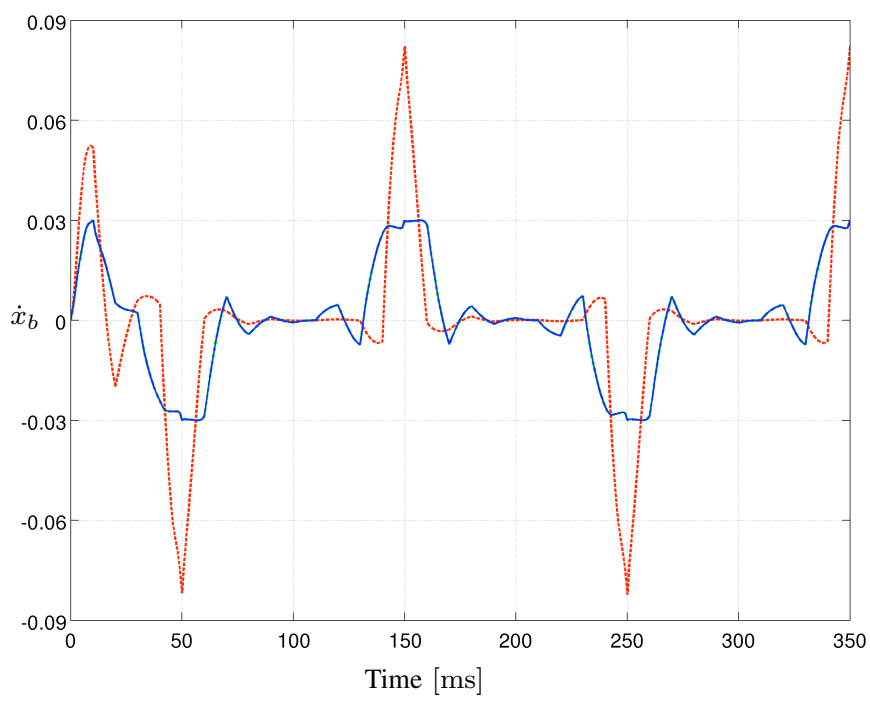

Fig. 5. Base-stage velocity $\dot{x}_{b}[\mathrm{~m} / \mathrm{s}]$, undisturbed. Constrained optimal periodic MPC (blue, solid). Unconstrained optimal periodic control (red, dashed). Repetitive-MPC (green, dash-dotted). Note constraint $\left|\dot{x}_{b}\right| \leq 0.03$.

solid black line in Fig. 4 denotes the reference trajectory. The optimal unconstrained control law has constraints on neither states nor inputs and achieves a very fast response, but at the expense of serious constraint violation in $\dot{x}_{b}$, and a little constraint violation in $x_{p}$. The optimal constrained periodic MPC control law achieves rigorous constraint satisfaction at the expense of a slightly sluggish response in $x_{p}$.

Next, a plant-model mismatch was included when performing simulations. Instead of the discrete-time dynamics of Eq. (1), for simulations the system $x_{i+1}=0.99\left(A x_{i}+B u_{i}\right)$ is employed, i.e. the state is forced to contract a little. Plotted in Fig. 6 is the piezo-stage position $x_{p}$. Three control laws are contrasted; the optimal constrained periodic MPC control law proposed in this paper (blue, solid), optimal

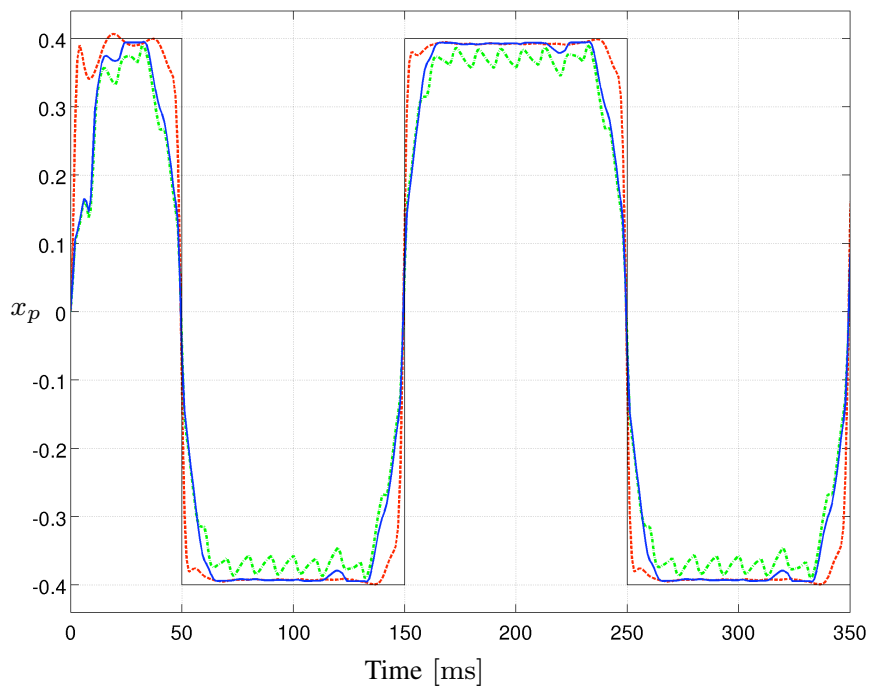

Fig. 6. Piezo-stage position $x_{p}[\mathrm{~mm}]$, disturbed. Constrained optimal periodic MPC (blue, solid). Unconstrained optimal periodic control (red, dashed). Repetitive-MPC (green, dash-dotted). Note constraint $\left|x_{p}\right| \leq 0.4$. 
unconstrained control based on periodic models and the solution of the periodic Riccati equation (red, dashed), and the RMPC strategy of [11] (green, dash-dotted). The solid black line denotes the reference trajectory. The optimal constrained periodic MPC and optimal unconstrained solutions do not track the reference as closely as in the nominal case plotted in Fig. 4, but have not changed significantly in any other way. The RMPC solution rigorously satisfies the constraints, but clearly performs very poorly compared to optimal constrained periodic MPC. The RMPC response has a larger error and is highly oscillatory. The oscillations are because the lifting to an LTI system reduces the effective sample-period to $0.1 \mathrm{kHz}$, and so the accumulated effects of the plant-model mismatch are only rejected at every 10th step. Note that in the case without plant-model mismatch plotted in Fig. 4, the RMPC response is identical to the optimal constrained periodic MPC response. This demonstrates how even small disturbances can cause severe performance degradation in RMPC, whereas the periodic MPC approach degrades more gradually with increasing disturbance levels.

\section{CONCLUSION}

Systems with periodically time-dependent state and control input dimension seem bizarre but elegantly model situations which are not. In this paper it was shown how linear time-invariant or linear periodic systems with multirate or multiplexed control inputs may be modeled as such systems. Periodic system models with time-dependent dimensions are lower dimensional, more general and more powerful than models with time-invariant dimensions, yet straightforward to deal with. The succinct and rigorous state-space modeling framework of linear periodic systems with possibly timedependent dimensions allowed state-feedback MPC theory for LTI systems with synchronous inputs to be straightforwardly extended to systems with unusual input timing constraints. Differences between state-feedback MPC theory for LTI systems and linear periodic systems are superficial, not conceptual. The solution of reverse periodic discrete-time algebraic Riccati equations with time-dependent dimensions is well studied. Definitions for (maximum) periodic controlled and positively invariant sets, and methods for their determination, were proposed here. Thus MPC problems for constrained linear periodic systems which result in infinitehorizon optimal state-feedback control laws were designed.

The periodic modeling framework with time-dependent dimensions is trivially extensible to modeling output-multirate (usually simply termed multirate) systems, which occur when multiple measurement rates induce cyclic time-dependence in outputs, or output-multiplexed systems, which occur when outputs are sampled at fixed time-intervals in ordered sequence (e.g. with polling sensors). Asynchronous outputs were ignore here because the discussion of MPC controller synthesis focused on state-feedback. The extension of outputfeedback MPC theory from the LTI case to the linear periodic case is of future research interest. Furthermore, the use of periodic systems to model asynchronous input or output behavior is readily applicable to control of nonlinear systems.

\section{REFERENCES}

[1] Albertos, P., Block Multirate Input-Output Model for Sampled-Data Control Systems, IEEE Trans. Aut. Control, Vol. 35, No. 9, pp. 10851088, 1990.

[2] Bittanti, S., Colaneri, P., \& De Nicolao, G., The Difference Periodic Riccati Equation for the Periodic Prediction Problem, IEEE Trans. Aut. Control, Vol. 33, No. 8, pp. 706-711, 1988.

[3] Blanchini, F., Set Invariance in Control, Automatica, Vol. 35, pp. 17471767, 1999.

[4] Böhm, C., Yu, S., \& Allgöwer, F., Predictive control for constrained discrete-time periodic systems using a time-varying terminal region, Proc. Conf. Methods and Models in Automation and Robotics, Poland, 2009.

[5] Chu, E. K. W., Fan, H. Y., Lin, W. W., \& Wang, C. S., Structurepreserving algorithms for periodic discrete-time algebraic Riccati equations, Int. J. Control, Vol. 77, No. 8, pp. 767-788, 2004.

[6] Colaneri, P., \& Longhi, S., The Realization Problem for Linear Periodic Systems, Automatica, Vol. 31, No. 5, pp. 775-779, 1995.

[7] Dória, C. E. T., \& Hennet, J. C., (A, B)-Invariant Polyhedral Sets of Linear Discrete-Time Systems, J. Optimization Theory and Applications, Vol. 103, No. 3, pp. 521-542, 1999.

[8] Gilbert, E. G., \& Tan, K. T., Linear Systems with State and Control Constraints: The Theory and Application of Maximal Output Admissible Sets, IEEE Trans. Aut. Control, Vol. 36, No. 9, pp. 1008-1020, 1991.

[9] Jones, C. N., \& Goncalves, J., A Cost-Effective Atomic Force Microscope for Undergraduate Control Laboratories, technical report, http://control.ee.ethz.ch/index.cgi?page= publications; action=details; id=3094, 2008.

[10] Kwon, W. H., \& Han, S., Receding Horizon Control: Model Predictive Control for State Models, Springer, ISBN 978-1846280245, 2005.

[11] Lee, J. H., Natarajan, S., \& , Lee, K. S., A model-based predictive control approach to repetitive continuous processes with periodic operations, J. Process Control, Vol. 11, pp. 195-207, 2001.

[12] Ling, K. V., Maciejowski, J. M., \& Wu, B. F., Multiplexed model predictive control, Proc. 16th IFAC World Congress, Czech Republic, 2005.

[13] Lovera, M., \& Varga, A., Optimal discrete-time magnetic attitude control of satellites, Proc. 16th IFAC World Congress, Czech Republic, 2005.

[14] Maciejowski, J. M., Predictive Control with Constraints, Pearson, ISBN 978-0-2013-9823-6, 2002.

[15] Mayne, D. Q., Rawlings, J. B., Rao, C. V., \& Scokaert, P. O. M., Constrained Model Predictive Control: Stability and Optimality, Automatica, Vol. 36, pp. 789-814, 2000.

[16] Ravi, R., Khargonekar, P. P., Minto, K. D., \& Nett, C. N., Controller Parameterization for Time-Varying Multirate Plants, IEEE Trans. Aut. Control, Vol. 35, No. 11, pp. 1259-1262, 1990.

[17] Richards, A., Ling, K. V., \& Maciejowski, J. M., Robust Multiplexed Model Predictive Control, Proc. 9th EUCA European Control Conference, Greece, 2007.

[18] Scattolini, R., \& Schiavoni, N., A Multirate Model-Based Controller, IEEE Trans. Aut. Control, Vol. 40, No. 6, pp. 1093-1097, 1995.

[19] Varga, A., Computational methods for periodic systems - An overview, Proc. 1st IFAC Workshop on Periodic Control Systems, Italy, 2001.

[20] Varga, A., Computation of Minimal Periodic Realizations of TransferFunction Matrices, IEEE Trans. Aut. Control, Vol. 49, No. 1, pp. 146-149, 2004.

[21] Varga, A., On solving discrete-time periodic Riccati equations, Proc. 16th IFAC World Congress, Czech Republic, 2005.

[22] Varga, A., An overview of recent developments in computational methods for periodic systems, Proc. 3rd IFAC Workshop on Periodic Control Systems, Russia, 2007.

[23] Vidal, R., Schaffert, S., Lygeros, J., \& Sastry, S., Controlled Invariance of Discrete Time Systems, HSCC LNCS 1790, Springer, 2000. 